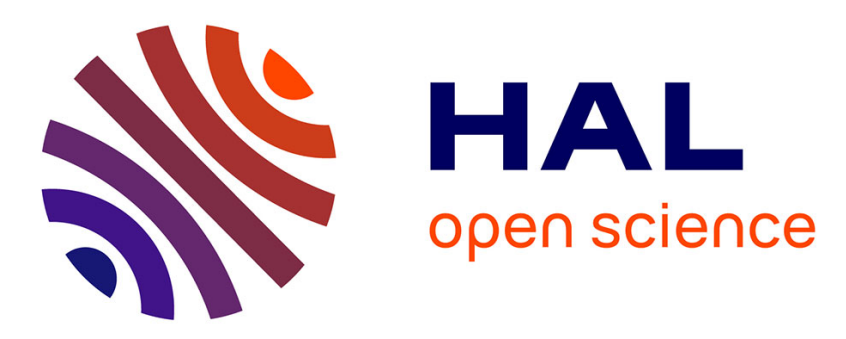

\title{
Enhanced ensemble-based 4DVar scheme for data assimilation
}

Yin Yang, Cordelia Robinson, Dominique Heitz, Etienne Mémin

\section{To cite this version:}

Yin Yang, Cordelia Robinson, Dominique Heitz, Etienne Mémin. Enhanced ensemblebased 4DVar scheme for data assimilation. Computers and Fluids, 2015, 115, 15 p. 10.1016/j.compfluid.2015.03.025 . hal-01144923

\section{HAL Id: hal-01144923 \\ https://hal.inria.fr/hal-01144923}

Submitted on 23 Apr 2015

HAL is a multi-disciplinary open access archive for the deposit and dissemination of scientific research documents, whether they are published or not. The documents may come from teaching and research institutions in France or abroad, or from public or private research centers.
L'archive ouverte pluridisciplinaire HAL, est destinée au dépôt et à la diffusion de documents scientifiques de niveau recherche, publiés ou non, émanant des établissements d'enseignement et de recherche français ou étrangers, des laboratoires publics ou privés. 


\title{
Enhanced ensemble-based 4DVar scheme for data assimilation
}

\author{
Yin Yang ${ }^{\mathrm{a}}$, Cordelia Robinson ${ }^{\mathrm{a}, \mathrm{b}}$, Dominique Heitz $^{\mathrm{b}}$, Etienne Mémin $^{\mathrm{a}}$ \\ ${ }^{a}$ Inria, Campus universitaire de Beaulieu, F-35042 Rennes Cedex, France \\ ${ }^{b}$ Irstea, UR TERE, F-35044 Rennes Cedex, France
}

\begin{abstract}
Ensemble based optimal control schemes combine the components of ensemble Kalman filters and variational data assimilation (4DVar). They are trendy because they are easier to implement than 4DVar. In this paper, we evaluate a modified version of an ensemble based optimal control strategy for image data assimilation. This modified method is assessed with a Shallow Water model combined with synthetic data and original incomplete experimental depth sensor observations. This paper shows that the modified ensemble technique is better in quality and can reduce the computational cost.
\end{abstract}

Keywords: Data assimilation, Optimal control, Ensemble methods, Shallow Water model, Depth sensor

\section{Introduction}

Data assimilation techniques aim at recovering the actual state of a system by combining the system's dynamics with noisy and partial measurements of this system. These techniques fulfill indeed a twofold objective. On the one hand, they provide a denoising - or reconstruction - procedure of the data through a given physical model, and on the other, they provide estimation procedures for the unknown parameters of the dynamics.

The literature offers a wide range of data assimilation techniques that can be divided into two main families. The first family ensues from stochastic filtering principles with the particle filter [1,2] and the Ensemble Kalman filter (EnKF) [3] as the principle representations. Both techniques are easy to implement regardless of the system's dynamics, however, this family suffers from a so-called "curse of dimensionality" due to the gigantic state space which has to be sampled with only very few samples for the sake of computational cost. The second family of data assimilation approaches, referred to as variational assimilation techniques, is formulated as an optimal control problem $[4,5]$. In this framework one seeks to estimate an optimal trajectory starting in the vicinity of a background solution and

Email addresses: iamyangyinegmail . com (Yin Yang), cordelia.robinson@inria.fr (Cordelia Robinson), dominique. heitz@irstea. fr (Dominique Heitz), etienne.memin@inria.fr(Etienne Mémin) leading to the lowest data discrepancy. Minimization procedures relying on the adjoint dynamics operator are required in this context. These methods are efficient from a computational point of view, however the construction and implementation of such adjoint model is often tedious and cumbersome in practice. This technique is classically referred to as 4DVar.

Recently, several schemes aiming at coupling the advantages of ensemble methods and variational assimilation strategies have been proposed. A first hybrid technique combining a fixed time variational data assimilation approach, called 3DVar, with an ensemble Kalman filter [6], was later extend to a hybrid technique with temporal variational data assimilation, i.e. 4DVar [7]. Several authors have proposed methods that express explicitly the solution as a linear combination of the square root of an empirical covariance constructed from an ensemble of samples [8, 9]. The optimization often relies on ensemble Kalman filter update and is sometimes coupled with an adjoint scheme [10].

In this work we chose to assess a method closely related to a strategy proposed in [11] and [12], which will be referenced as 4DEnVar in the following. This technique introduces in its objective function an empirical ensemble-based background-error covariance that avoids the building of the tangent linear and adjoint model of the dynamics. The associated optimization is conducted as a gradient descent procedure and does not rely on iterative ensemble filtering updates exploiting equivalences between Kalman smoothers and the $a$ 
posteriori energy minimization established only in the linear case [13]. An experimental evaluation based on a shallow water model and Kinect depth sensor image sequences was carried out in this study. Results of the proposed 4DEnVar methods were compared with those given by a classical 4DVar approach.

\section{4D Variational Data Assimilation}

Variational data assimilation technique aims at estimating the best fit trajectory between the background trajectory, determined by a dynamical model from a prior initial state $X_{0}^{b}$, and the measurements $y$ of the system. In practice, the dynamics operator involved is nonlinear and the variational assimilation procedure can be improved by introducing a nonlinear least squares procedure in the same spirit as a Gauss-Newton incremental strategy [14]. In this strategy, the dynamics is linearized around the background trajectory and the optimization is performed in terms of an optimal increment $\delta X$. The assimilation system reads

$$
\begin{aligned}
& \partial_{t} \delta X(t, x)+\partial_{X} \mathbb{M}(X) \delta X(t, x)=0, \\
& \delta X\left(t_{0}, x\right)=X_{0}^{b}-X_{0}+\eta \\
& \mathcal{Y}(t, x)=\mathbb{H}\left(\varphi_{t}\left(X_{0}(x)\right)\right)+\epsilon(t, x),
\end{aligned}
$$

where $\partial_{X} \mathbb{M}(X)$ denotes the tangent linear operator of the dynamics operator $\mathbb{M}$. $X_{0}^{b}$ is an arbitrary background condition, $X_{0}$ is the initial guess and $\eta$ is some error of background covariance $B$. In the third equation,

$$
\varphi_{t}\left(X_{0}(x)\right)=X_{t}=X_{0}+\int_{0}^{t} \mathbb{M}\left(x_{s}\right) d s
$$

denotes the flow map. It depends only on a (possibly random) initial condition. The state variables $X_{t}$ and the measurements $\mathcal{Y}$ are linked by an observation operator $\mathbb{H}$, up to an observation error $\epsilon$ assumed to be a zero mean i.i.d. (independent and identically distributed) Gaussian random field with covariance tensor $R$. In this study, for simplification purpose, we will consider a linear observation operator set to the identity or to an incomplete identity operator when only a part of the state is observable.

The optimal increment $\delta X_{0}$ at time $t_{0}$ is obtained by minimizing the following objective function reads

$$
\begin{aligned}
J\left(\delta X_{0}\right) & =\frac{1}{2}\left\|\delta X_{0}(x)\right\|_{B}^{2}+ \\
\frac{1}{2} & \int_{t_{0}}^{t_{f}}\left\|\partial_{X} \mathbb{H} \delta X(t, x)-D(t, x)\right\|_{R}^{2} d t,
\end{aligned}
$$

where the innovation vector $D(t, x)$ is defined as

$$
D(t, x)=\mathcal{Y}(t, x)-\mathbb{H}\left(\varphi_{t}\left(X_{0}(x)\right)\right) .
$$

The objective function involves the $L_{2}$-norm with respect to the inverse covariance tensor $\|f\|_{A}^{2}=$ $\int_{\Omega} f(x) A^{-1}(x, y) f(y) d x d y$.

The incremental variational data assimilation naturally leads to an algorithm with two nested loops. The outer loop computes the flow map trajectory, $\varphi_{t}\left(X_{0}(x)\right)$, through the nonlinear dynamics, while the internal loop computes an optimal increment driven by the tangent linear dynamics with respect to the trajectory.

It is possible to define different variants of this strategy depending on the point used for the linearization $[14,15]$. In both approaches, a fixed background error covariance matrix is used for the entire minimization process over successive outer loops. In this paper, we want to face typical cases involved in remote sensing where only bad quality backgrounds are available through noisy images with possibly large areas of missing data. Hence, we advocate for a slightly different approach. Since the background state is poorly known, the background error covariance approximated at the beginning of one assimilation process should not be used for additional outer loops. Indeed, we propose to update this error covariance matrix (since this error is actually calculated based on the increments produced by previous inner loop, it is no longer the actual background error). This strategy will be elaborated in the next section combined with the preconditioning technique.

\subsection{Standard incremental 4DVar assimilation with the adjoint approach}

In order to minimize the objective function (5), we need to cancel the gradient $\partial_{\delta X} J$. This comes to solve the following linear system:

$$
\begin{array}{r}
\left(B^{-1}+\int_{t_{0}}^{t_{f}} \partial_{X} \varphi_{t}^{*} \partial_{X} \mathbb{H}^{*} R^{-1} \partial_{X} \mathbb{H} \partial_{X} \varphi_{t} d t\right) \delta X_{0}= \\
\int_{t_{0}}^{t_{f}} \partial_{X} \varphi_{t}^{*} \partial_{X} \mathbb{H}^{*} R^{-1} D(t, x) d t,
\end{array}
$$

where the left hand operator is the Hessian matrix, denoted as $\mathcal{H}$. Its expression involves the adjoint operators $\partial_{X} \varphi_{t}^{*}$ and $\partial_{X} \mathbb{H}^{*}$. Due to the dimension of the state space, the minimization requires an iterative optimization strategy. An elegant solution to this problem consists in relying on an adjoint formulation [4]. Within this formalism, the gradient functional is obtained by a forward integration of the dynamical system followed by a backward integration of an adjoint variable, $\lambda$, driven by 
1. Set an initial state: $X\left(t_{0}\right)=X^{b}\left(t_{0}\right)$

2. From $X\left(t_{0}\right)$, compute $X(t)$ with the forward integration of the nonlinear dynamics (4)

3. Set the initial increment as: $\delta X_{0}=0$

4. Compute the tangent linear dynamics (1)

5. Compute the adjoint variable $\lambda(t)$ with the backward integration of relation (8)

6. Update the initial value $\delta X\left(t_{0}\right)$ with (9)

7. Loop to step 4 until convergence

8. Update the initial condition: $X\left(t_{0}\right)+:=$ $\delta X\left(t_{0}\right)$

9. Loop to step 2 for a specified number of iterations

Figure 1: Schematic representation of the incremental variational data-assimilation algorithm

the linear tangent of the dynamics' operator. The adjoint system is defined as:

$$
\left\{\begin{array}{l}
-\partial_{t} \lambda(t)+\left(\partial_{X} \mathbb{M}\right)^{*} \lambda(t)= \\
\left(\partial_{X} \mathbb{H}\right)^{*} R^{-1}\left(D(t, x)-\partial_{X} \mathbb{H} \partial_{X} \varphi_{t}\left(X_{0}\right) \delta X_{0}\right) \\
\lambda\left(t_{f}\right)=0
\end{array}\right.
$$

and the functional gradient at the initial time is given by,

$$
\partial_{\delta X} J\left(\delta X_{0}\right)=-\lambda\left(t_{0}\right)+B^{-1} \delta X_{0}
$$

A schematic algorithm representation of the overall 4DVar assimilation is provided in figure 1.

\subsection{Preconditioning of the 4DVar assimilation}

The possibly ill-conditioned nature of system (7) depends on the condition number of the Hessian matrix. The larger the condition number, the more sensitive the system with respect to errors in the estimate and the slower the inner loop convergence rate [16]. A preconditioning of the variational incremental system consists in applying a change of variable with the matrix square-root of the background error covariance matrix $B$. This transformation called the control variable transform (CVT) reads

$$
\delta X_{t}=B^{\frac{1}{2}} \delta Z_{t}
$$

and leads to a new Hessian matrix $\widetilde{\mathcal{H}}=B^{\frac{1}{2} T} \mathcal{H} B^{\frac{1}{2}}$ possessing a lower condition number [16]. As for the choice of the preconditioning matrix, [17] introduced the use of $B$ as the preconditioning matrix and associated bi-conjugate gradient method (BiCG) as the minimizing technique. In addition, they proposed the employment of a truncated whole Hessian as the preconditioning matrix and argued that it can achieve faster convergence.

Applying the CVT (10) to the function (5), we get a modified objective function,

$$
\begin{aligned}
J\left(\delta Z_{0}\right) & =\frac{1}{2}\left\|\delta Z_{0}\right\|^{2}+ \\
& \frac{1}{2} \int_{t_{0}}^{t_{f}}\left\|\partial_{X} \mathbb{H} \partial_{X} \varphi_{t}\left(X_{0}\right) B^{\frac{1}{2}} \delta Z_{0}-D(t, x)\right\|_{R}^{2} d t .
\end{aligned}
$$

This modified cost function removes $B^{-1}$ from the background term. Hence no cross-variable correlation is anymore involved in the control vector $\delta Z_{0}$. Note this change of variable can also be viewed as a whitening filtering of the background error. Despite a better conditioning, the resulting system remains in general difficult to solve and requires the use of the adjoint minimization setups.

Let us note that ideally we should use the actual background error covariance matrix to precondition the system. However, in our case, this matrix is badly known due to a noisy reference. Instead of using a fixed model to formulate the background matrix, we suggest to use an empirical approximation of this covariance matrix defined from the current solution (Eq. (12)).

$$
B^{k}=\overline{\left(X_{0}^{k-1}-{\overline{X_{0}}}^{k-1}\right)\left(X_{0}^{k-1}-{\overline{X_{0}}}^{k-1}\right)^{T},}
$$

where $k$ is the outer loop index and the operator $\overline{f(t)}$ takes the mean value of $f(t)$ and $X_{0}^{0}=X_{0}^{b}$. We assume this approximation is sufficiently close to the actual covariance matrix to have $\left(B^{k}\right)^{-1} B \approx \mathbb{I}$. This approximation becomes better and better along the succession of external loops. In the following we show how to set a low rank approximation of this matrix through an ensemble of realizations. Since we are dealing with the preconditioned form of the cost function, this successive approximation of the background error covariance matrix actually constitutes a change of the preconditioning matrix $B^{\frac{1}{2}}$ shown by Eq. (10). Note that such a change of the preconditioning matrix only effects the subspace to which the increments belong. Similar to the original algorithm proposed in [14], the whole convergence of the minimization toward a global minimum is not guaranteed. 


\section{Ensemble-based 4DVar}

\subsection{Low rank approximation of the background error covariance matrix}

The ensemble-based 4D variational assimilation scheme is defined within the framework of preconditioned incremental variational system (11) while handling an empirical approximation of the background covariance matrix [8, 11]. This low rank approximation of the background covariance matrix is directly inspired from the Ensemble Kalman filter where the covariance terms are estimated from the spread of an ensemble of samples. Denoting $\langle f(t)\rangle=N^{-1} \sum_{1}^{N} f^{(i)}(t)$ as an empirical ensemble mean of a quantity $f(t)$ through $N$ samples, the empirical background covariance matrix is

$$
B \approx \frac{1}{N-1} \sum_{i=1}^{N}\left(X^{(i), b}-\left\langle X^{b}\right\rangle\right)\left(X^{(i), b}-\left\langle X^{b}\right\rangle\right)^{T} .
$$

Noting $A_{b}^{\prime}:=\frac{1}{\sqrt{N-1}}\left(X_{0}^{(1) b}-\left\langle X_{0}^{b}\right\rangle, \ldots, X_{0}^{(N) b}-\left\langle X_{0}^{b}\right\rangle\right)$, the perturbation matrix gathering the $N$ zero mean centered background ensemble members as a low-dimensional approximation of the background matrix. Introducing the background covariance approximation in the preconditioned cost function (11), we get

$$
\begin{aligned}
J\left(\delta Z_{0}\right) & =\frac{1}{2}\left\|\delta Z_{0}\right\|^{2}+ \\
\frac{1}{2} & \int_{t_{0}}^{t_{f}}\left\|\partial_{X} \mathbb{H} \partial_{X} \varphi_{t}\left(X_{0}\right) A_{b}^{\prime} \delta Z_{0}-D(t, x)\right\|_{R}^{2} d t .
\end{aligned}
$$

The whole term $\partial_{X} \varphi_{t}\left(X_{0}\right) A_{b}^{\prime}$ corresponds to a forecast by the dynamical model of the centered square-root background covariance matrix. As we rely here on an empirical description of this matrix from a set of samples, we can observe that integrating these samples in time provides us immediately an empirical expression of a low-rank approximation of the background covariance trajectory and of its square root. This avoids thus the employment of the adjoint operator. We set hence:

$$
\widetilde{B}_{t}^{\frac{1}{2}}=\partial_{X} \varphi_{t}\left(X_{0}\right) A_{b}^{\prime} .
$$

The gradient of the cost function is now given by:

$$
\partial_{\eta} J=\delta Z_{0}+\int_{t_{0}}^{t_{f}} \widetilde{B}_{t}^{\frac{1}{2} T}\left(\partial_{X} \mathbb{H}\right)^{*} R^{-1}\left(\partial_{X} \mathbb{H} \widetilde{B}_{t}^{\frac{1}{2}} \delta Z_{0}-D(t, x)\right) d t .
$$

and its Hessian is:

$$
\widetilde{\mathcal{H}}=\mathbb{I}+\int_{t_{0}}^{t_{f}} \widetilde{B}_{t}^{\frac{1}{2} T}\left(\partial_{X} \mathbb{H}\right)^{*} R^{-1} \partial_{X} \mathbb{H} \widetilde{B}_{t}^{\frac{1}{2}} d t
$$

Once the minimizer $\widehat{\delta Z}_{0}$ estimated, the analysis reads:

$$
X_{0}^{a}=X_{0}^{b}+{\widetilde{A_{b}^{\prime}}}_{\delta^{\delta}} .
$$

Let us emphasize that, as the covariance matrix $\widetilde{B}$ is at most of rank $N-1$, the control variable has at most $N-1$ non null components in the eigenspace. Compared to the full 4DVar approach, the control variable's degrees of freedom are thus considerably lowered and the minimization computational complexity is significantly decreased. Indeed, this ensemble version has a lower computation cost if the ensemble forecasting step is distributed on a grid computing.

\subsection{Preconditioning Matrix Update}

As mentioned earlier, in this study we focus on situations where the background state is only poorly known. It is hence essential to allow in the estimation process a substantial deviation from the background state. So unlike typical incremental ensemble-based variational methods which keep a fixed background covariance and apply a single outer loop of the Gauss-Newton minimization, we propose to update the approximation of this associated error covariance between two consecutive outer loops. The update of the error covariance can be either derived from the ensemble of analysis based on perturbed observations or by a direct transformation of the background ensemble perturbations. The first method relies on a perturbed ensemble of observations, generated with an additional noise with the same standard deviation than in equation (3):

$$
\boldsymbol{y}^{j}=\mathcal{Y}+\epsilon^{j}, \quad j=1, \ldots, N .
$$

At the $k$ th outer loop iteration, the innovation vector of the $j$ th member of the initial ensemble $X_{0}^{(j), k}$ is defined as,

$$
D^{(j), k}(t, x)=\mathcal{Y}^{j}(t, x)-\mathbb{H}\left(\varphi_{t}\left(X_{0}^{(j), k}\right)\right), \quad j=1, \ldots, N .
$$

with $X_{0}^{(j), 0}=X_{0}^{(j), b}$. Thus a parallel realization of minimization with regard to each member of initial ensemble is conducted,

$$
\begin{aligned}
& \delta Z_{0}^{(j), k}=\left(A_{b}^{\prime k}\right)^{-1} \delta X_{0}^{(j), k}, \quad j=1, \ldots, N . \\
& J\left(\delta Z_{0}^{(j), k}\right)=\frac{1}{2}\left\|\delta Z_{0}^{(j), k}\right\|^{2}+ \\
& \frac{1}{2} \int_{t_{0}}^{t_{f}}\left\|\partial_{X} \mathbb{H} \partial_{X} \varphi_{t}\left(X_{0}\right) A_{b}^{\prime k} \delta Z_{0}^{(j), k}-D^{(j), k}(t, x)\right\|_{R}^{2} d t .
\end{aligned}
$$


Finally the updated initial ensemble field and its perturbation matrix read:

$$
\begin{aligned}
& X_{0}^{(j), k+1}=X_{0}^{(j), k}+A_{b}^{\prime k}{\widehat{\delta Z_{0}}}^{(j), k}, \quad j=1, \ldots, N . \\
& A_{b}^{\prime k+1}=\frac{1}{\sqrt{N-1}}\left(X_{0}^{(1), k+1}-\left\langle X_{0}^{k+1}\right\rangle, \ldots, X_{0}^{(N), k+1}-\left\langle X_{0}^{k+1}\right\rangle\right) .
\end{aligned}
$$

The direct transformation approach corresponds to a linear transformation of the initial error's $\left(X_{b}-X_{t}\right)$ covariance. This approach can take many forms as the transformation matrix is not unique, here we opt for a mean preserving transformation as used in Ensemble Transform Kalman filter, the updated background ensemble perturbations reads,

$$
A_{b}^{\prime k+1}=A_{b}^{\prime k}\left\{\mathbb{I}+\int_{t_{0}}^{t_{f}} \widetilde{B}_{t}^{\frac{1}{2} T}\left(\partial_{X} \mathbb{H}\right)^{*} R^{-1} \partial_{X} \mathbb{H} \widetilde{B}_{t}^{\frac{1}{2}} d t\right\}^{-\frac{1}{2}, k} \mathbf{V}
$$

It corresponds to the Hessian square root computed from previous perturbation matrix at outer loop iteration $k{ }^{1}$ As the minimization algorithm LBFGS relies on an approximation of the inverse Hessian matrix $\mathcal{H}^{-1}$, we can use this byproduct to evaluate equation (23). At the initial time, the background matrix is fixed from the initial random conditions chosen. The arbitrary orthogonal matrix $\mathbf{V}$ is used to center the posterior ensemble on the updated initial condition/analysis. In this approach a single minimization process is conducted with respect to the background state in opposition to previous cases where the minimization has to be done with respect to each member of the ensemble plus the background state. Finally the updated initial ensemble fields are,

$$
X_{0}^{(j) k+1}=\widehat{X}_{0}^{k}+\sqrt{N-1} A_{b}^{\prime(j) k+1}, \quad j=1, \ldots, N
$$

where $\widehat{X}_{0}^{k}$ corresponds to the updated initial state at the $k$ th outer loop,

$$
\widehat{X}_{0}^{k}=X_{0}^{k}+A_{b}^{\prime k}{\widehat{\delta Z_{0}}}^{k}
$$

Both variants of the update will be assessed in the experimental section.

\subsection{Localization Issues}

The previous ensemble method relies on a low rank approximation of the background matrix. This empirical approximation built from only very few samples,

\footnotetext{
${ }^{1}$ Note that the relationship between the Hessian and the covariance matrix holds rigorously in a linear sense; in a nonlinear scenario, this relationship is only an approximation.
}

compared to the state space dimension, leads in practice to spurious correlations between distant points. For ensemble Kalman techniques, it is customary to remove these long distance correlations through localization procedure. There are generally two methods to filter the pseudo-correlations.

The first approach introduces a Schur element-wise product between the background correlation matrix and a local isotropic correlation function : $P_{b}=C \odot B$. The spatial correlation function can be simply defined as a matrix $C(\|x-y\| / L)$ in which we set $C_{x y}=0$ when the distance between $x$ and $y$ exceeds the cutoff distance $L$. Polynomial approximations of a Gaussian function with compact support and a hard cutoff are often employed [18] to that end. They lead to sparse correlation matrices, which is computationally advantageous. In order to incorporate the localized background error matrix into our system, we approximate the square root of $P_{b}$ by a spectral decomposition of the isotropic correlation function and keep only the $r$ first leading Fourier modes

$$
C^{\prime}=E_{n \times r} \lambda_{r \times r}^{1 / 2}
$$

The modified perturbation matrix is then provided by

$$
P_{b}^{\prime}=\left(\operatorname{diag}\left(\mathbf{A}_{b}^{\prime(1)}\right) C^{\prime}, \ldots, \operatorname{diag}\left(\mathbf{A}_{b}^{\prime(N)}\right) C^{\prime}\right) .
$$

Here the diag operator sets the vector $X_{b, k}^{\prime}$ as the diagonal of a matrix. This localized perturbation matrix is used to precondition the assimilation system associated with (14), (15) and (16). Remark that this approach is incompatible with the deterministic update of background error covariance matrix. This is due to the inconsistency of matrix dimensions when updating the background ensemble based on Eq.(24). As the dimension of $P_{b}^{\prime}$ is $n \times N \times r$ instead of $n \times N$, the ensemble perturbation matrix can not be recovered from its localized counterpart.

A variant of covariance localization approach based on [7] is implemented in [19] along with other localization schemes. [19] also highlights the importance of the dimension of the control vector which is directly related to the cost of the minimization algorithm.

Another localization technique proposed by [20] employs local ensemble. This approach involves a transformation $\mathbb{M}_{l}$ from state space $\mathbb{R}^{n}$ to local space $\mathbb{R}^{l}$, the local vector is defined as:

$$
X_{l}=\mathbb{M}_{l} X_{n},
$$

Then the analysis process is done in local space $\mathbb{R}^{l}$ around each grid point only incorporating the model points and observations within a certain range. This 
certain range, denoted as $l$, which corresponds to the concept of cut-off distance aforementioned, determines the size of local space. This localization strategy is ideally compatible with the method of direct transformation approach associated with the update of background error covariance matrix. The great advantage of this combination is its low computational cost when implemented with properly parallelized minimization procedures. However, good performances can only be reached with a small local space.

All these elements (i.e. CVT, localization and incremental/background error covariance matrix update) associated with a LBFGS minimization strategy constitute the proposed ensemble method. The algorithm descriptions of the overall methods are presented in figure 2 and 3. We point out that this assimilation system-composed of perturbed observations, one outer loop and localization via a modified covariance matrix- is equivalent to the 4DEnVar method proposed by $[11,21]$. If direct ensemble transformation update is used, it is hence close to the 4D-LETKF method [9] and the IEnKS method [22] but with a minimization performed on a variational basis.

\section{Experimental evaluation and comparison}

\subsection{Flow configuration and comparison tools}

We chose to assess our method with both synthetic and experimental observations with analogous setups. The simulated and real experiments carried out in this study consisted in observing the free surface of a fluid contained in a rectangular flat bottom tank of size $L_{x} \times$ $L_{y}=250 \mathrm{~mm} \times 100 \mathrm{~mm}$. More specifically we observed the evolution of a unidirectional wave generated by an initial height difference $\Delta h$ of the free surface as illustrated in figure 4 . In the following the characteristic velocity $U$ is considered as an approximation of wave phase velocity $\sqrt{g \Delta h L_{x} / \sqrt{L_{x}^{2}+\Delta h^{2}}}$ due to $\Delta h$ the initial difference of maximum and minimum surface height.

RMSE analysis. The Root Mean Square Error (RMSE) is a way of measuring of the differences between values predicted by a model or an estimator and the values actually observed. The RMS between a predicted state $X^{f}$ and the observed state $X^{\text {obs }}$ is defined by:

$$
\mathrm{RMSE}=\frac{1}{n} \sqrt{\sum_{i=1}^{n}\left(X^{f}-X^{o b s}\right)^{2}},
$$

\section{Ensemble-based variational data assimilation algorithm: : Localize covariance approach}

1. Set an initial state: $X\left(t_{0}\right)=X^{b}\left(t_{0}\right)$ and the ensemble $X_{0}^{j, b}$ as an arbitrary choice (for the 1 st cycle) or as the forecast state and the ensemble forecast derived from the previous assimilation cycle respectively

2. Define matrix $\mathbf{X}(t)=\left[X\left(t_{0}\right), X_{0}^{1, b}, \ldots, X_{0}^{N, b}\right]$ concatenating the initial ensemble and compute in parallel $\mathbf{X}(t)$ with the forward integration of the nonlinear dynamics (1)

3. Generate ensemble observations (19) and innovations (20)

4. Derive the background perturbation matrix localization technique from (27)

5. Initialize the increment matrix: $\delta \mathbf{X}_{0}$ : $\left[\delta X_{0}, \delta X_{0}^{(1)}, \ldots, \delta X_{0}^{(N)}\right]$

6. Do an inverse control variable transformation $\delta \mathbf{R}_{\mathbf{0}}=\left(P_{b}^{\prime}\right)^{-1} \delta \mathbf{X}_{0}$ if necessary where $\left(P_{b}^{\prime}\right)^{-1}$ is calculated by SVD

7. Optimize in parallel $\delta \mathbf{R}_{\mathbf{0}}$ in the inner loop, the cost function and the gradient are calculated based on the modified versions of (21)

8. Update control initial space $\mathbf{R}_{\mathbf{0}}$ and calculate $\delta \mathbf{X}_{0}$ by transforming $\delta \mathbf{R}_{\mathbf{0}}$ to the state space with (10)

9. Update the initial ensemble and ensemble perturbation matrix (22)

10. Loop to step 2 for a specified number of iterations

11. Evolve the analysis state $\mathbf{X}^{\mathbf{a}}\left(\mathbf{t}_{\mathbf{0}}\right)$ to the beginning of the next cycle through the nonlinear dynamics (1). The forecast state and forecast ensemble are used to initialize the next assimilation cycle.

Figure 2: Schematic representation of the complete ensemble based incremental variational data assimilation algorithm with localize covariance approach 
Ensemble-based variational data assimilation algorithm: Local ensemble approach

1. Similar to steps 1 and 2 in the algorithm 2

2. Define local space and the transformation from state space to local space (28)

3. Parallelizing minimization computation at each grid point $(p, q)$

4. Derive the background ensemble perturbation covariance $A_{b}^{\prime}$, initialize the increment vector $\delta Z_{0}$, and do an inverse control variable transformation but in the local space around grid point $(p, q)$

5. The cost function minimization is done only once in terms of the background. The updated background is obtained by (25)

6. Update the initial ensemble and ensemble perturbation matrix (24 and 23)

7. Reconstruct the analysis and the ensemble form local space to state space

8. Similar to steps 10 and 11 in the algorithm 2

Figure 3: Schematic representation of the complete ensemble based incremental variational data assimilation algorithm with local ensemble approach

When we deal with the synthetic data, we will base our analysis on the comparison of the background, observation and assimilated states' RMSE with respect to the true solution. Furthermore, the observations time step is many times bigger than the numerical time step, thus we will be able to assess the results on a very small amount of observations. We will also consider the RMSE comparison on a semilogarithmic graph.

\subsection{Dynamical model}

Within this context, the flow was well described by the following shallow water model relating the flow height $h$ and the free surface velocity field $(u, v)^{T}$

$$
\begin{cases}\partial_{t} h+\partial_{x}(h u)+\partial_{y}(h v) & =0 \\ \partial_{t}(h u)+\partial_{x}\left(h u^{2}\right)+\partial_{y}(h u v)+\frac{1}{2} g \partial_{x} h^{2} & =0 \\ \partial_{t}(h v)+\partial_{x}(h u v)+\partial_{y}\left(h v^{2}\right)+\frac{1}{2} g \partial_{y} h^{2} & =0\end{cases}
$$

where $g$ is the standard gravity and where the Coriolis force and the bottom friction were neglected. The system of equations (30) was solved in a computational domain $L_{x} \times L_{y}=5 \Delta h \times 2 \Delta h$ discretized on square grid of $n_{x} \times n_{y}=101 \times 41$ points. Finite volume numerical schemes were used to evaluate all spatial derivatives [23]. Time integration was performed with a third-order
Runge-Kutta scheme. A fixed time step was used equal to $\Delta t U / L_{x}=0.003$ to ensure the Courant-FriedrichLevy condition. The no-slip condition at the boundaries was imposed directly via a Dirichlet boundary condition.

The tangent and adjoint models corresponding to the numerical shallow water model and necessary for the 4DVar approach were constructed with the automatic differentiation tool Tapenade [24].

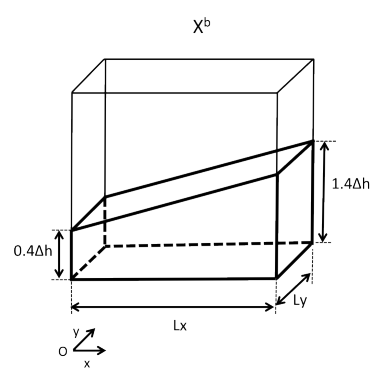

(a)

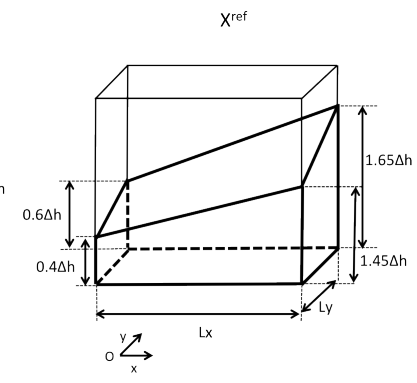

(b)
Figure 4: Initial free surface heights to simulated trajectories of (a) the background and (b) the reference free surface.

To evaluate the performance of the proposed approaches we carried out twin experiments with simulated observations from the shallow water model described in $\S 4.2$. Hence in these experiments, the model used to create the simulated data was the same as the model used for the assimilation. However, the initial conditions used to generate the background trajectory were different from the initial conditions used for the reference trajectory. The initial background height field was fixed as a smooth slope tilted along the $x$-axis by $20 \%$ and the initial background velocity field was fixed as zero. Whereas for the reference trajectory, the initial reference height was a smooth slope tilted along the $x$-axis and $y$-axis by respectively $21 \%$ and $10 \%$, and the initial reference velocity field was fixed as a Gaussian field with a standard deviation of $1 \mathrm{~mm} / \mathrm{s}$. Figure 4 illustrates the initial surface heights used to simulate background and reference trajectories. Here the initial height difference was equal to $\Delta h=20 \% L_{x}=50 \mathrm{~mm}$ and the characteristic wave velocity was equal to $U=$ $694 \mathrm{~mm} / \mathrm{s}$.

The synthetic observations were generated by adding i.i.d. Gaussian noise perturbations to the reference free surface's height and velocity fields at each grid points and every 50 time steps. A single assimilation window containing five observations uniformly distributed in time was adopted. The assimilation trajectory lasted 
$0.895 L_{x} / U$

In order to construct the background error covariance matrix, we used different strategies with respect to the incremental 4DVar and the group of ensemble-based variational techniques. For the incremental 4DVar, we adopted a static diagonal matrix $B=\sigma_{b}^{2} \mathbb{I}$, where $\sigma_{b}$ was optimally tuned as the standard deviation between the true solution and the background initial state. For the 4DEnVar, as the background error covariance is derived from the ensemble fields, it is crucial that the initial ensemble represents correctly the background errors. Here, if no other suffix is indicated, the default case in the figures is the Gaussian perturbation approach. This approach with zero mean Gaussian error is nevertheless biased and the bias must be estimated as well [25]. Considering that the reference solution deviates strongly from the a priori configuration, we experimented a parameter perturbation strategy, consisting in defining the initial members from a random drawing of different free surface height slopes. This approach is indicated by the "PP" suffix in the figures. We also highlighted the method of [11] (indicated by "Liu-et-al" suffix in figures) using perturbed observations, a single outer loop and localized covariances. Other proposed strategies with several outer loops, localized covariances or local ensemble are indicated by suffix "OL", "LC" and "LE" respectively. Note that the initial state (perturbed surface height and null velocity field), was integrated for a few time steps before we started the assimilation process. This provided us the guaranty of balanced velocity perturbations that complied well with the nonlinear dynamic model.

The assimilation techniques were first evaluated with partially observed systems where only the free surface height was available, and then with a fully observed system, i.e. with height and velocities. The RMSE curves corresponding to these two cases are gathered in figures $5 \mathrm{a}$ and $5 \mathrm{~b}$. In both cases, the ensemble technique was efficient when the initial noise complied with the physics of the observed phenomenon (i.e. random slopes drawing-para versus gaussian noise). As indicated in table 1, this configuration was also the fastest. For non-physical initial noise, the localization is mandatory and the cutoff distance was set as $20 \% L_{x}$ (the same value was used to define the size of local space). For partially observed system, the 4DEnVar assimilation technique globally led to better results compared to the standard 4DVar. The initial free surface was strongly corrected in both methods, however the unobserved velocity components were well corrected only for the ensemble technique. For this peculiar configuration where only the free surface height was observed,
Table 1: Comparisons of the CPU time $(2 \times 2.66 \mathrm{GHz}$ QuadCore Intel Xeon) and memory demands (16 GB in total) with $10^{5}$ level of state size between different methods.

\begin{tabular}{lcc} 
& CPU Time $[\mathrm{s}]$ & Memory \\
\hline 4DVar & 3200 & Small \\
4DEnVar-PP $(\mathrm{N}=8$, No Localization $)$ & 120 & Small \\
4DEnVar-LC $(\mathrm{N}=32)$ & 2400 & Huge \\
4DEnVar-LE $(\mathrm{N}=32)$ & 600 & Small
\end{tabular}

three general remarks can be drawn. First, 4DVar results could be improved with a better description of the background error covariance matrix. Moreover, several outer loops clearly improved the results highlighting the pertinence of the background covariance update. Finally, the method with local ensemble yielded slightly better results than the method with localized covariance.

For a fully observable system, the advantage of ensemble methods were less notable than in the previous case. In that context, the impact of background covariance matrix design was weaker. This might be due to the fact that each state variable was mainly corrected by the corresponding measurements rather than indirectly from other observed components. With a low number of samples, the LE approach gave better results than the LC approach. When increasing the sample numbers, the difference between the two localization approaches diminished. This can be explained by the local space used in the LE method that was already rather small compare to the full state space, so the curse of dimensionality effect induced by few ensemble numbers was less severe in this case. The computational time of the different methods is indicated in table 1. The parameter perturbation approach provided the best performances in terms of CPU time and memory demanding, which was expected as no localization was applied. Nevertheless, it should be noted that when considering real observations, finding the compatible parameter perturbation formalism corresponding to initial error statistics is not a trivial task. Between the two localization techniques, the cost of LE approach was lower but with expected limitations. The local space was restricted to a small size as increasing the local space led to both quadratic increasing computation cost and worse localization. On the other hand, the LC approach maintained a nearly constant computational cost in terms of the cut-off distance despite its rather high CPU time and huge memory appetite.

\subsection{The experimental case}

We carried out another evaluation on a real world experiment in which the free surface of a fluid contained 
in a rectangular flat bottom tank of size $L_{x} \times L_{y}=$ $250 \mathrm{~mm} \times 100 \mathrm{~mm}$ was observed. Following the work of $[26,27]$, we used the Kinect sensor to observe the evolution of a unidirectional wave generated by an initial free surface height difference $\Delta h=1 \mathrm{~cm}$ on a grid of $222 \times 88$ pixels. The flow surface was located between 680 and $780 \mathrm{~mm}$ from the device. When the attenuation coefficient of the liquid is larger than $113 \mathrm{~m}^{-1}$, the Kinect sensor displays a mean measurement error of 0.5 $\mathrm{mm}$ with standard deviations of about $0.5 \mathrm{~mm}$ for both flat and sinus-like surfaces. The sensor captures successfully sinus-like varying elevations with spatial periods smaller than $20 \mathrm{~mm}$ and amplitudes smaller than $2 \mathrm{~mm}$. In the following the characteristic velocity $U$ is considered as an approximation of wave phase velocity $\sqrt{g \Delta h}$.

In terms of the observation errors: for a point in the unobserved region, we set the observation error as a function of the distance from the closest observed point. Thus, the longer the distance, the larger the error. The observation error is however bounded by a maximal value of $60 \%$ of the height difference $\Delta h$. Within the observed region, we set the observation error homogeneously to the instrument error $\sigma_{o}=0.5 \% \Delta h$.

In this case, the initial background was completely unknown hence, it was set to a filtered observation with interpolated values on the missing data regions at the initial time on a $248 \times 98$ grid. The observed free surface behaved roughly as an unidirectional wave along the $\mathrm{x}$ axis. Thus, we set the initial velocity field as a smooth linear slope with a velocity at the top of the wave equal to $23 \%$ of the wave velocity $U$, and a null velocity at the bottom of the wave.

The assimilation scheme was adapted to sliding assimilation windows to avoid long range temporal correlations. Five windows over nine observations in times were adopted, each window consisted in five observations and started successively form the 1 st up to the 5th observation. The lengthy of a single window is chosen to fit 5 observations in order to keep consistency with the synthetic case. Similarly to the synthetic case, the assimilation started at the second image in order to construct balanced ensemble through the integration between the two first images of a set of members. Those ensemble members are defined beforehand by adding to the background state a Gaussian perturbation fields with standard deviation $\sigma_{b}$. In addition, the localized covariance technique requires the preset of the cutoff distance before proceeding the assimilation process. Both of these parameters have been calibrated through a sensitivity analysis. Figure 6 presents the evolution of the cost function final value with respect to $\sigma_{b}$ and to the cut-off distance while the other parameters (e.g. ensemble members, window length etc.) were kept fixed. The plot shows that the cost function decreased with $\sigma_{b}$. In addition we observed that the numerical model instability increased with $\sigma_{b}$. That led to an optimal range for $\sigma_{b}$ in $[3.6 \% \Delta h, 14.4 \% \Delta h]$. The best compromise within this interval was $\sigma_{b}=3.6 \% \Delta h$.

Likewise, the optimal cutoff distances belonged to the interval $\left[10 \% L_{x}, 20 \% L_{x}\right]$. Any value which was larger than $20 \% L_{x}$ or smaller than $10 \% L_{x}$ could be detrimental to the system. This range is reasonable since longer cutoff distances were inclined to in-corporate spurious long correlations while shorter cutoff distance could result in an underestimation of the error covariance. Note that such sensitivity study provides only rough indications as these optimal values are subject to change if other conditions of the assimilation system vary. Finally we chose $\sigma_{b}=3.6 \% \Delta h$, the cutoff distance as well as the size of local space were fixed as $15 \%$ of the length $L_{x}$.

The results obtained by both assimilation techniques are displayed in figure 8 in terms of the averaged wave crest surface height as function of the time. This is quite intuitive as we were dealing here with a single wave simulation. Thus, we are particularly interested in the wave crest's region rather than other quite flat regions. We observed that the 4DVar and the 4DEnVar could both follow the observation trajectory tendency. While the 4DVar tended to underestimate the surface height at the beginning of the assimilation window, the group of 4DEnVar yielded very similar results between the first and fourth image. After the fifth image, the result of 4DEnVar by Liu et al. diverged from the observation trajectory.

We also compared the free surface height distribution in figure 7. According to these free surfaces, we can see that the 4DVar solution showed some difficulties to handle the discontinuities at the boundaries of the regions in which the data have been extrapolated. Discontinuities in the 4DVar solution between the observed regions and the very noisy region appeared clearly. The 4DEnVar provided much more satisfying results on the boarders. They were smoother and corresponded clearly to a better compromise between the observation and the model. An alternative approach using the limited-memory bundle method (LMBM) to deal with non-smooth observation operators is proposed in [28] with implementing code file available in [29].

\section{Conclusions and perspectives}

In this paper we proposed and evaluated the performance of an enhanced ensemble-based optimal control 
strategy for noisy situation such as the one encountered in image data assimilation. Comparisons between a standard 4DVar technique and two ensemble-based 4DVar methods were carried out with a shallow water model and with simulated and real observation image sequences.

The proposed ensemble-based methods show great potential in handling incomplete and noisy observations. With partial state variable observations, the ensemble technique outperformed the standard 4DVar at reconstructing the unobserved components with a lower computational cost. With very noisy and spatially incomplete observations, the ensemble methods offered better physical consistent background error covariances. Both advantages make ensemble methods a perfect tool to assimilate image data. Results outlined the efficiency of a physical noise compared to non physical noise requiring a localization procedure.

In a future work, we will apply this ensemble-based 4DVar method to directly assimilate SST (Sea Surface Temperature) image data with a surface quasigeostrophic (SQG) model. We will also introduce a stochastic shallow-water model [30], since in the framework of image assimilation the resolution of observation space is normally quite high. The use of a stochastic model could be computationally more advantageous by carrying out the dynamics on coarser grids.

\section{References}

[1] N. Gordon, D. Salmond, A. Smith, Novel approach to non-linear/non-Gaussian Bayesian state estimation, IEEE Processing-F 140 (2).

[2] A. Cuzol, E. Mémin, A stochastic filtering technique for fluid flow velocity fields tracking, IEEE Tran. on Pattern Analysis and Machine Intelligence 31 (7) (2009) 1278-1293.

[3] G. Evensen, Sequential data assimilation with a non linear quasi-geostrophic model using Monte Carlo methods to forecast error statistics, J. Geophys. Res. 99 (C5) (10) (1994) 143-162.

[4] F.-X. Le Dimet, O. Talagrand, Variational algorithms for analysis and assimilation of meteorological observations: theoretical aspects, Tellus A 38A (1986) 97-110.

[5] A. Gronskis, D. Heitz, E. Mémin, Inflow and initial conditions for direct numerical simulation based on adjoint data assimilation, Journ. Comp. Phys 242 (6) (2013) 480-497.

[6] T. M. Hamill, C. Snyder, A Hybrid Ensemble Kalman Filter-3D Variational Analysis Scheme, Mon. Wea. Rev. 128 (8) (2000) 2905-2919.

[7] A. C. Lorenc, The potential of the ensemble Kalman filter for NWP - a comparison with 4D-Var, Quart. J. Roy. Meteor. Soc. 129 (2003) 3183-3203.

[8] M. Buehner, Ensemble-derived stationary and flow-dependent background-error covariances: Evaluation in a quasioperational NWP setting, Quart. J. Roy. Meteor. Soc. 131 (607) (2005) 1013-1043.

[9] E. J. Fertig, J. Harlim, B. R. Hunt, A comparative study of 4DVAR and a 4D Ensemble Kalman Filter: perfect model simulations with Lorenz-96, Tellus A 59 (1) (2007) 96-100.
[10] A. M. Clayton, A. C. Lorenc, D. M. Barker, Operational implementation of a hybrid ensemble/4D-Var global data assimilation system at the Met Office, Quart. J. Roy. Meteor. Soc. 139 (675) (2012) 1445-1461.

[11] C. Liu, Q. Xiao, B. Wang, An Ensemble-Based FourDimensional Variational Data Assimilation Scheme. Part I: Technical Formulation and Preliminary Test, Mon. Wea. Rev. 136 (9) (2008) 3363-3373.

[12] M. Buehner, P. L. Houtekamer, C. Charette, H. L. Mitchell, B. He, Intercomparison of Variational Data Assimilation and the Ensemble Kalman Filter for Global Deterministic NWP. Part I: Description and Single-Observation Experiments, Mon. Wea. Rev. 138 (5) (2010) 1550-1566.

[13] Z. Li, M. Navon, Optimality of 4D-Var and its relationship with the Kalman filter and Kalman smoother, Quart. J. Roy. Meteor. Soc. 127 (572) (2001) 661-684.

[14] P. Courtier, J.-N. Thépaut, A. Hollingsworth, A strategy for operational implementation of 4D-Var, using an incremental approach, Quart. J. Roy. Meteor. Soc. 120 (519) (1994) 13671387.

[15] A. T. Weaver, J. Vialard, D. L. T. Anderson, Three- and FourDimensional Variational Assimilation with a General Circulation Model of the Tropical Pacific Ocean. Part I: Formulation, Internal Diagnostics, and Consistency Checks, Mon. Wea. Rev. 131 (2003) 1360-1378.

[16] S. A. Haben, A. S. Lawless, N. K. Nichols, Conditioning and preconditioning of the variational data assimilation problem, Comput Fluids 46 (1) (2011) 252-256.

[17] A. El Akkraoui, Y. Trémolet, R. Todling, Preconditioning of variational data assimilation and the use of a bi-conjugate gradient method, Quart. J. Roy. Meteor. Soc. 139 (672) (2012) 731741.

[18] G. Gaspari, S. E. Cohn, Construction of correlation functions in two and three dimensions, Quart. J. Roy. Meteor. Soc. 125 (554) (1999) 723-757.

[19] G. Desroziers, J.-T. Camino, L. Berre, 4DEnVar: link with 4D state formulation of variational assimilation and different possible implementations, Quart. J. Roy. Meteor. Soc. 140 (2014) 2097-2110.

[20] E. Ott, B. R. Hunt, I. Szunyogh, A. V. Zimin, M. C. E. J. Kostelich, E. Kalnay, D. J. Patil, J. A. Yorke, A local ensemble Kalman filter for atmospheric data assimilation, Tellus A 56A (2004) 415-428.

[21] C. Liu, Q. Xiao, B. Wang, An Ensemble-Based FourDimensional Variational Data Assimilation Scheme. Part II: Observing System Simulation Experiments with Advanced Research WRF (ARW), Mon. Wea. Rev. 137 (5) (2009) 16871704.

[22] M. Bocquet, P. Sakov, An iterative ensemble Kalman smoother, Quart. J. Roy. Meteor. Soc. 140 (682) (2013) 1521-1535.

[23] S. F. Bradford, B. F. Sanders, Finite-volume model for shallowwater flooding of arbitrary topography, J. Hydraul. Eng. 128 (2002) 289-298.

[24] L. Hascoët, V. Pascual, The Tapenade Automatic Differentiation tool: Principles, Model, and Specification, ACM Transactions On Mathematical Software 39 (3) (2013)

[25] D. P. Dee, Bias and data assimilation, Quart. J. Roy. Meteor. Soc. 131 (613) (2005) 3323-3343.

[26] B. Combès, A. Guibert, E. Mémin, D. Heitz, Free-surface flows from Kinect: Feasibility and limits, in: FVR2011 - Forum on recent developments in Volume Reconstruction techniques applied to 3D fluid and solid mechanics, Univ. Poitiers, Futuroscope Chasseneuil, France, 2011.

[27] B. Combes, D. Heitz, A. Guibert, E. Mémin, A particle filter to reconstruct a free-surface flow from a depth camera, Fluid 
Dynamics ResearchMinor revision.

[28] J. L. Steward, I. M. Navon, M. Zupanski, N. Karmitsa, Impact of non-smooth observation operators on variational and sequential data assimilation for a limited-area shallow-water equation model, Quart. J. Roy. Meteor. Soc. 138 (663) (2011) 323-339.

[29] J. L. Steward, I. M. Navon, M. Zupanski, N. Karmitsa, Compressed file implementing code for the paper "Impact of nonsmooth observation operators on variational and sequential data assimilation for a limited-area shallow-water equation model" in QJRMS (2014).

[30] E. Mémin, Fluid flow dynamics under location uncertainty, Geophys. Astrophys. Fluid Dyn. 108 (2) (2014) 119-146. 

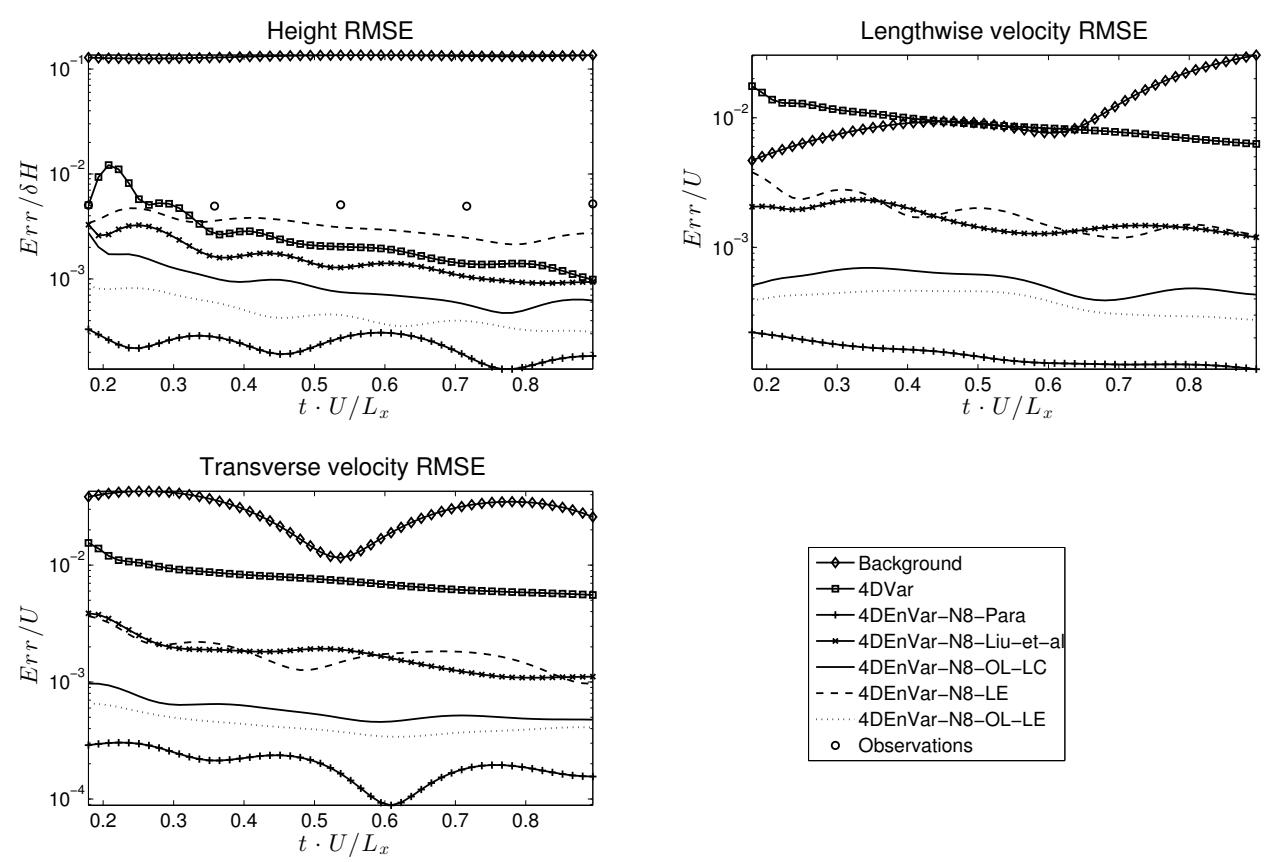

(a)
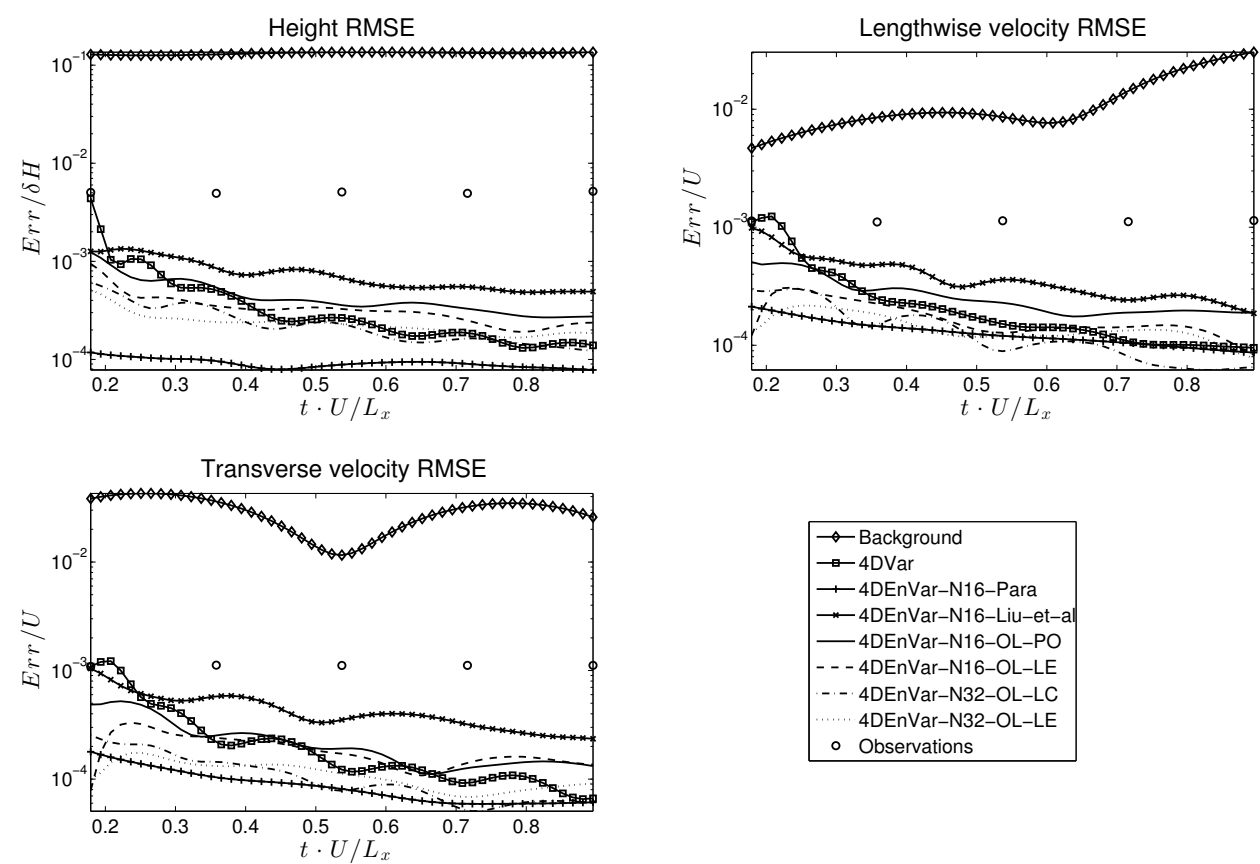

(b)

Figure 5: RMSE comparisons between an incremental 4DVar and 4DEnVar assimilation approaches for two types of observations: (a) partially observed system through noisy free surface height; (b), fully observed system (i.e. free surface height and velocity fields). 

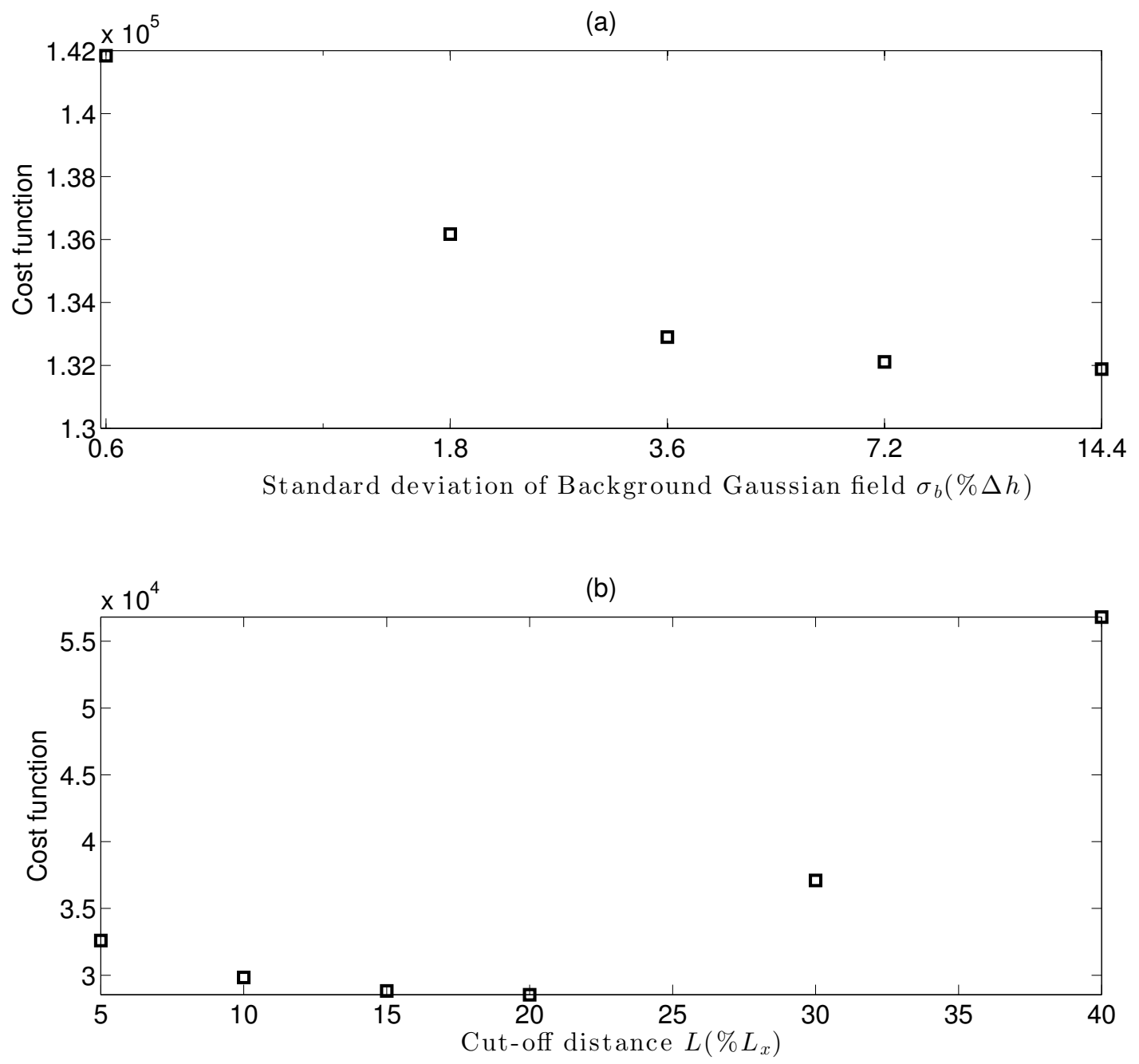

Figure 6: Sensitivity analysis of the standard deviation of the background Gaussian perturbation filed (a) and the cut-off distance (b). 

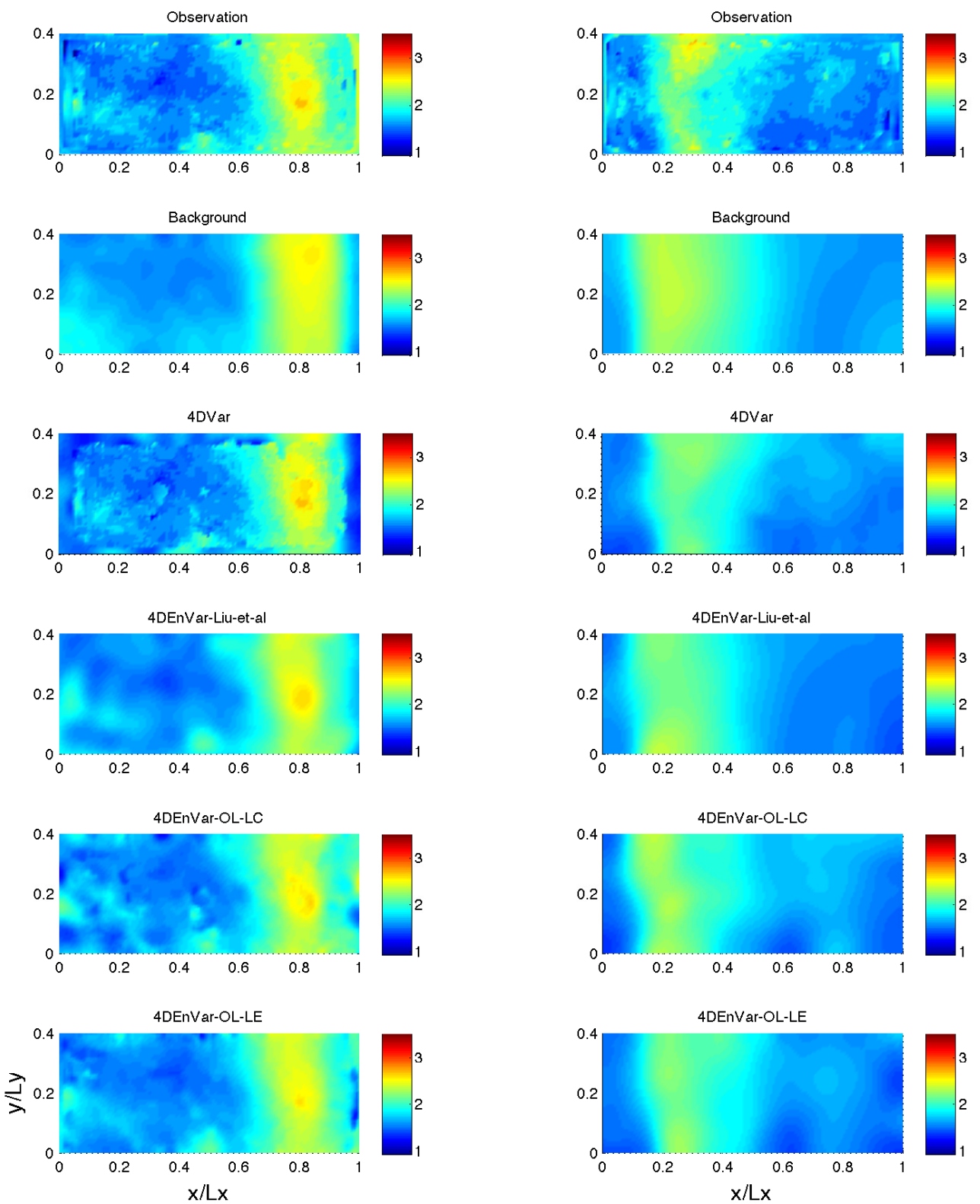

Figure 7: Comparisons of height fields $h / \Delta h$ colormaps for from top to bottom: Observations, Background, 4DVar, 4DEnVar-Liu-et-al, 4DEnVarOL-LC, 4DEnVar-OL-LE; left column at $t \cdot U / L_{x} \simeq 0.065$, right column at $t \cdot U / L_{x} \simeq 0.586$. 


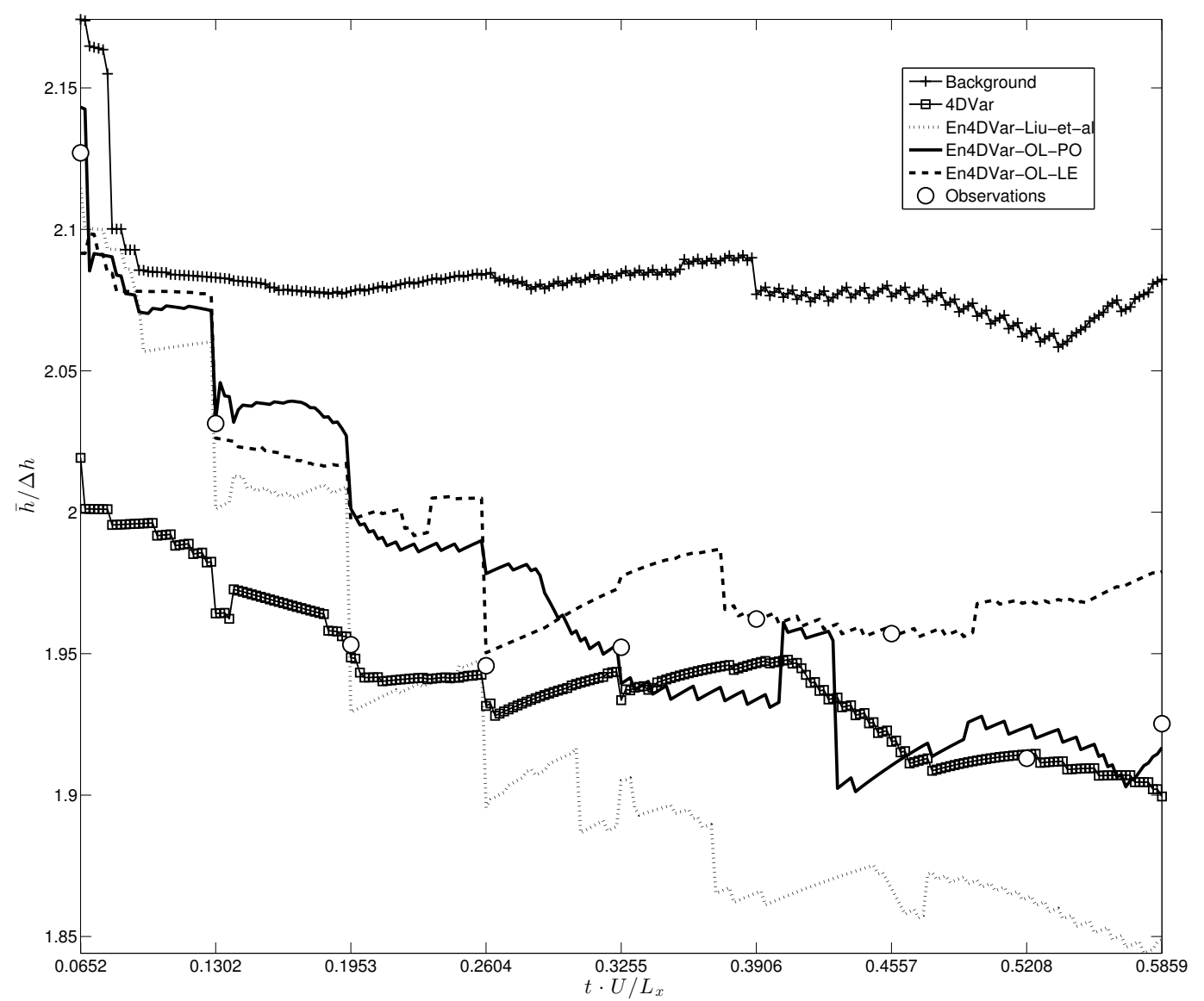

Figure 8: Mean surface height of the wave crest region as a function of time - comparisons of different variational data assimilation approaches results. 